\title{
Isoniazid Preventive Therapy Implementation among People Living with HIV/AIDS Enrolled in Care at Specialist Hospital Yola, Nigeria
}

\author{
Article by Kabiru Usman Suru ${ }^{1}$, J E Chiegil ${ }^{1}$, S O Adeyemi ${ }^{1}$, O F Martins ${ }^{2}$ \\ ${ }^{1}$ Texila American University Guyana, \\ ${ }^{2}$ Department of Public Health, Federal Medical Centre Yola, Adamawa State, Nigeria \\ E-mail: kabirusuru@yahoo.com ${ }^{1}$
}

\begin{abstract}
Background: Nigeria ranks $5^{\text {th }}$ among the 22 countries that contributed $80 \%$ of the global Tuberculosis (TB) burden. The risk of developing TB is between 20 and 37 times greater in people living with HIV (PLWHIV) than among those who do not have HIV infection. The purpose of this study was to assess the level of Isoniazid Preventive Therapy (IPT) implementation among PLWHIV.

Methods: Retrospective cohort study conducted to examine the IPT implementation among the PLWHIV in Specialist Hospital Yola, Nigeria. Information's were extracted from the IPT enrolment register and client's folders, analyzed with excel and compared with the data in the District Health Information System (DHIS). Chi-square test was used to measure the strength of associations between the various variables and probability of statistical significant level set $<0.05$ at $95 \%$ Confidence interval

Results: Eight hundred and seventy-three (873) clients were enrolled in to HIV care and treatment from January-December 2015 and were all screened for TB. 777 (89\%) clients had no presumptive TB, only 273 (35\%) clients were initiated on IPT out of those without presumptive TB. 143 (93\%) clients on ART completed their IPT as against the 97 (80\%) Pre-ART clients that completed IPT.

Conclusion: The IPT utilization in the prevention of TB among the PLWHIV in this health facility was very low, however consistent availability of Isoniazid and effective adherence counselling to eligible PLWHIV before commencement of IPT would help in increasing uptake and reducing the rate of defaulters on IPT.
\end{abstract}

Keywords: Tuberculosis, Implementation, Isoniazid Preventive Therapy, PLWHIV, Nigeria.

\section{Introduction}

Tuberculosis (TB) is one of the major opportunistic infections contributing to high morbidity and mortality among HIV sero-positive persons. (WHO 2015) The HIV pandemic has resulted in the increase cases of tuberculosis affecting the most economically productive age group, especially in sub-Saharan Africa countries. This condition is worsened by poverty, inadequate case detection, diagnosis and treatment, poorly financed health systems and the deepening impact of HIV pandemic. (WHO 2007). The risk of developing TB is between 20 and 37 times greater in people living with HIV (PLWHIV) than among those who do not have HIV infection. (Nwokeukwu H I, et al., 2015). TB is responsible for more than a quarter of deaths in people living with HIV (WHO 2011). In 2012, of 8.6 million people who developed TB, 1.1 million were HIV infected; 320,000 deaths from HIV-associated TB accounted for approximately a quarter of all HIV-associated deaths. (WHO 2013). TB remains the leading preventable cause of morbidity and mortality in PLHIV. If not adequately addressed, TB has the potential to cause great set back in the effort made globally in rapidly expanding HIV care and treatment. Therefore, prevention of TB is one of the most important measures needed to reduce morbidity and mortality among PLHIV, especially in countries with a high TB and HIV burden like Nigeria. (Melissa A.B. et al. 2015).

There has been increase spread of HIV in the past few decades, particularly in sub-Saharan Africa, this has been accompanied by a major increase in the number of new cases of tuberculosis [Global Fund to Fight AIDS, TB and Malaria. 2012]. In 2010, TB killed an estimated 1.68 million people, including 0.38 
DOI: $10.21522 / \mathrm{TIJPH} .2013 .05 .04 . \mathrm{Art} 022$

ISSN: $2520-3134$

million deaths among TB patients who were HIV positive (UNAID 2012). The proportion of TB and HIV co-infection is highest in African countries. Overall, the African region accounted for $82 \%$ of TB cases among PLWHIV (WHO 2011). According to the global fund to fight AIDS, TB and malaria (GFATM) information note, the interaction between TB and HIV presents additional challenges to TB control. It is imperative to improve and strengthen TB/HIV collaborative activities to reduce the burden of TB in PLHIV and reduce the burden of HIV among TB patients (GFATM. 2012). Collaborative TB/HIV management is essential to ensure that HIV positive TB patients are identified and treated appropriately, and to prevent TB in HIV positive people. Activities to decrease the burden of TB among HIV patients include intensified TB case finding (ICF), for those without active TB, INH preventive therapy (IPT) and infection control (IC) in health-care and congregate settings. (Denegetu and Dolamo, 2014).

Isoniazid Preventive therapy (IPT) is the use of Isoniazid in HIV Positive individuals with latent TB infection and under five contacts of PTB index cases to prevent the development of active TB disease. Available evidence shows that TB is the commonest opportunistic infection and the leading cause of death among People living with HIV/AIDS (PLWHA) and that IPT is effective in preventing this ugly incidence [FMOH 2008]. Despite the availability of policies and recommendations and significant evidence that IPT reduces incidence of TB in HIV-infected individuals, the utilization of IPT is still lower than expected. The uptake and delivery of IPT is still slow in most African countries. Lack of standard operating procedures, guidelines and screening algorithms, lack of health care provider capacity in providing the service, and shortage of preventive therapy supplies contributes to the low availability and poor uptake of IPT (Granich R, et al., 2010).

Research evidence shows that IPT markedly reduces the incidence of TB among people living with HIV and even among those receiving ART. WHO and UNAID in a 1998 statement issued on the effectiveness of IPT recommended its use as part of essential care package for people living with HIV. It recommends IPT for all PLWHIV in areas with a prevalence of latent TB infection $>30 \%$ and for all people with documented latent TB infection or exposure to an infectious TB case, regardless of where they live. (Nwokeukwu H I, et al., 2015). Although TB represents a potential threat to the achievable benefits of HIV scale up care and treatment, IPT represents strong potential for reduction of mortality and morbidity in people living with HIV. Therefore, major effort should be made to identify, prevent and treat TB among people living with HIV enrolled in to HIV care and treatment.

Many health care workers have expressed concerned about isoniazid (INH) resistance due to the possible failure to rule out a diagnosis of an active TB, and this serve as a major barrier to IPT implementation in most of our health centers. Failure to rule out a diagnosis of an active TB might be due to inexperienced staff or other organizational barriers. Findings from a systematic review have suggested that the effect of IPT on isoniazid resistance is likely to be small. Other concerns raised include the limited durability of isoniazid's protective benefit and the need to provide adherence support to achieve high rates of adherence and treatment completion (Howard and El-Sadr 2010). Studies have also suggested that clinical opinion leaders could be instrumental in changing fears about INH resistance (Chehab J C, et al, 2011).

The findings of Assebe and his group showed that IPT use was associated with fifty percent reduction in new cases of tuberculosis and probability of developing TB was higher in non-IPT group (Assebe et al, 2015). Thus, implementing the widespread use of IPT has the potential to reduce TB rates substantially among HIV-infected individuals in addition to other tuberculosis prevention and control effort in resource limited settings. (Assebe et al, 2015) Despite these evidence, implementation of IPT in HIV care and treatment programs in resource-constrained environments has been limited.

The findings of this study will help the Adamawa state government in Nigeria to find out the rate of IPT implementation among the PLWHIV in Adamawa State, barriers affecting it implementation and areas that need to be addressed so that the incidence of TB can be reduce among the PLWHIV. It will also provide the state government and other Donor agencies supporting the HIV care and treatment services in the State with information on the level of IPT implementation and the way forward. 


\section{General objective}

To assessed the level of IPT implementation among PLWHIV in Specialist hospital Yola, Adamawa state, Nigeria.

\section{Specific objectives}

- To determine the proportion of PLHIV attending HIV care clinics who were screened for TB.

- To determine the proportion of PLHIV without presumptive TB placed on IPT during their follow-up cares.

- To determine the level of adherence to IPT among the HIV positive Patients.

- To come up with recommendation on how to improve IPT implementation in Adamawa State

\section{Methods}

Study Type: A retrospective cohort study, designed to assessed the level of IPT implementation among the PLWHIV in Specialist Hospital Yola, Nigeria (January -Dec 2015).

Study Setting: This study was conducted in Specialist Hospital Yola which is the first referral public hospital in Adamawa State, rendering referral and specialize medical services to more than 3 million populations in Adamawa State. The hospital is one of the secondary health facilities designated to render HIV care, support and treatment in Adamawa State with the support of USAID since 2007. The hospital has enrolled more than 10,000 patients to HIV care and follow-up, among these about 4,000 were on preART care follow-up during the study period.

Study Population: The source population was HIV infected individuals enrolled in HIV care and treatment. The study population was a sample of the source population who were under care during the period of January to December 2015. Exclusion criteria were incomplete patient records (HIV infected persons with unknown IPT start and end date), past history of TB, active TB, or on treatment for TB. All patients who were on the IPT register for January-Dec 2015 were included in the analysis.

Sampling frame: We used the patient's folders and IPT enrolment register.

Sampling Size Determination and Sampling Procedure: The required sample size was determined using single population proportion formula $\mathrm{n}=\left(\mathrm{z}^{2} * \mathrm{P}(1-\mathrm{P}) / \mathrm{d}^{2}\right)$,

where $\mathrm{n}$ is the desired sample size (when population is greater than 10,000).

$\mathrm{z}$ is the standard normal score set at 1.96 ,

$\mathrm{d}$ is the desired degree of accuracy set at 0.05 .

$\mathrm{p}$ is the estimated proportion of the target population. By taking $\mathrm{p}=50 \%$.

$\mathrm{n}=\mathrm{z}^{2} *(1-\mathrm{P}) / \mathrm{d} 2$

$\mathrm{n}=(1.96)^{2} *(0.05)(0.05) /(0.05)^{2}$

$\mathrm{n}=384$

The computed sample size was 384. (Margaret O A. 2004)

To eliminate the design effect, the sample size was doubled and $14 \%$ of the total sample size was added as Contingency so that to accommodate the total number of clients enrolled during the study period. Hence, the total sample size used in this study was $(384 \times 2)+14 \%=873$.

Sampling Procedure, Data Collection and Analysis: Information was extracted from the IPT enrolment register and clients' folders and analyzed with excel. To cross check for correctness and completeness the information was compared with the data that have been captured into the District Health Information System (DHIS) and Lafiya Management Information system (LAMIS) which are electronic medical records information storing system use in the hospital. Chi-square test was used to measure the strength of associations between the various variables and probability of statistical significant level set $<0.05$ at $95 \%$ Confidence interval.

Ethical Considerations: Ethical clearance was obtained from the Adamawa State Health Research Ethics Committee. This approval was communicated to the Head of ART unit and Head of the Monitoring and evaluation unit of the hospital. 
DOI: $10.21522 / \mathrm{TIJPH} .2013 .05 .04 . \mathrm{Art} 022$

ISSN: $2520-3134$

\section{Results}

From the study, a total of Eight hundred and seventy-three (873) clients were enrolled in to HIV care and treatment from January to December 2015. All of them were screened for TB which represent one hundred percent $(100 \%)$ of the total number of the patients enrolled in to care and treatment. Of these, four hundred and thirty (430) were male and four hundred and forty-three (443) were females. Out of the 873 clients that were screened for TB 777 (89\%) were without presumptive TB and 273 (35\%) were initiated on IPT. 


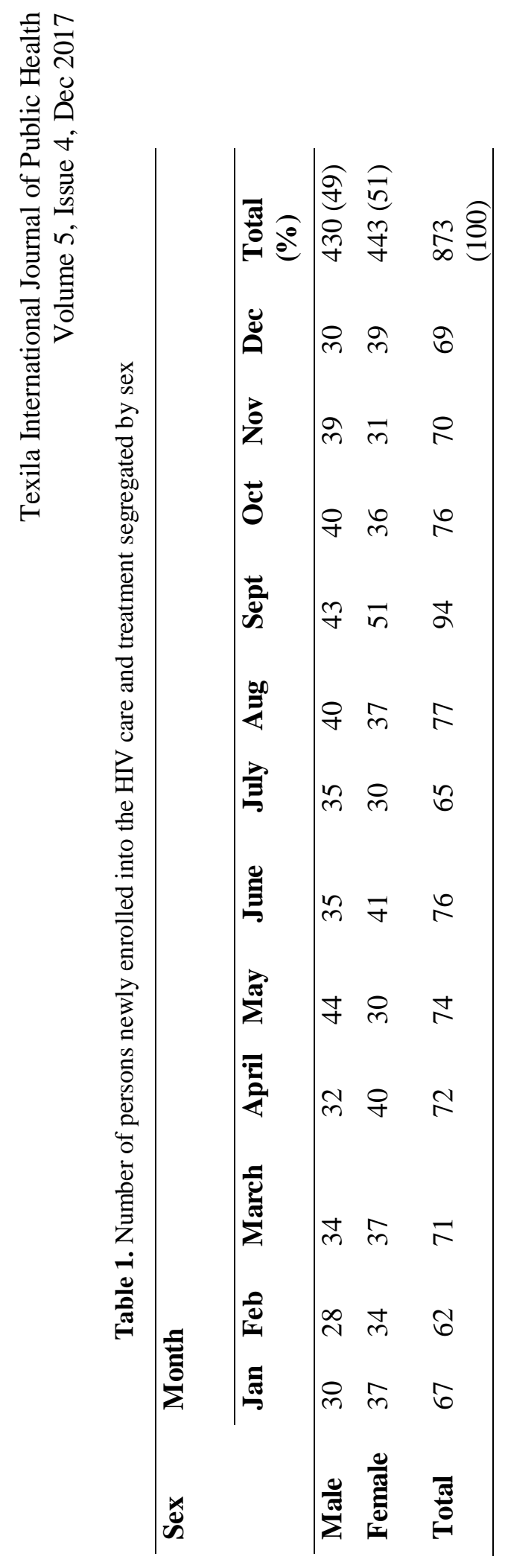


DOI: $10.21522 / \mathrm{TIJPH} .2013 .05 .04 . \mathrm{Art} 022$

ISSN: $2520-3134$

The table above shows that during the review period fourty nine percent of the total clients enrolled in to the HIV care and treatment were male while fifty one percent were female.

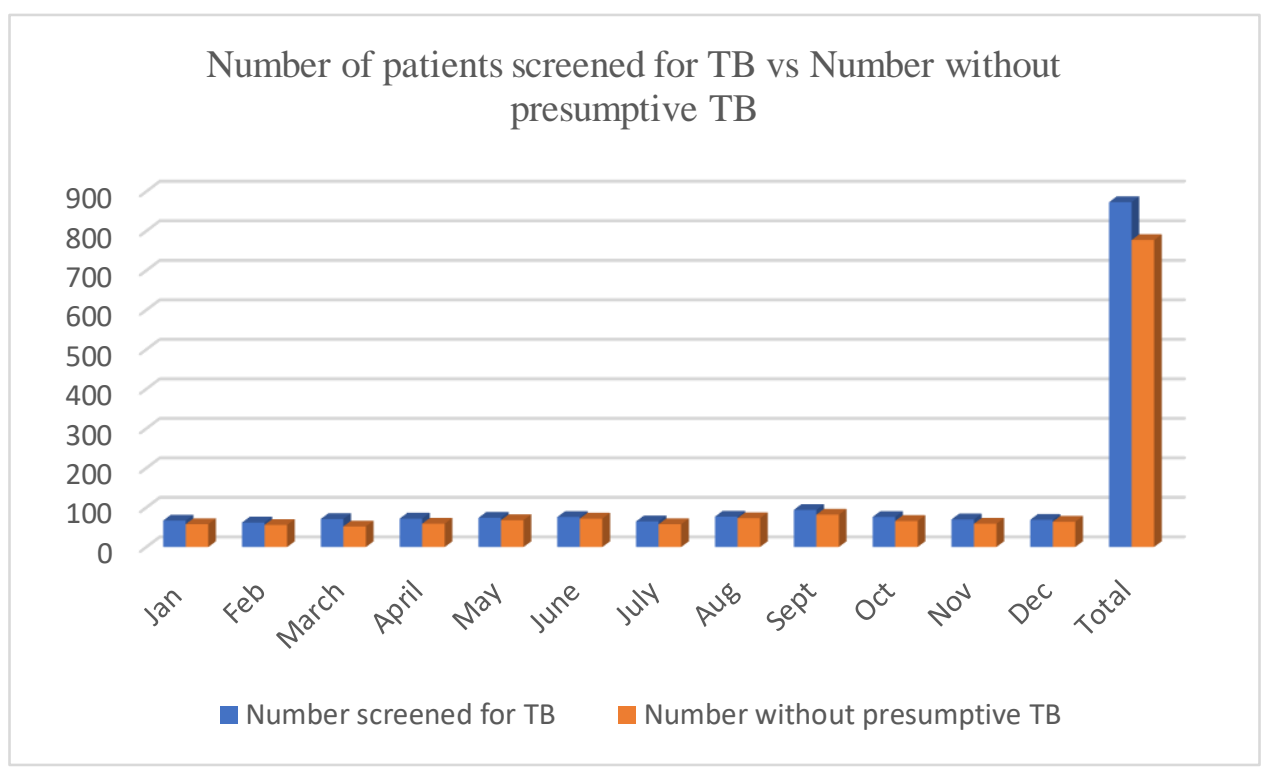

Figure 1. No of newly enrolled clients screened for TB Vs number without presumptive TB 


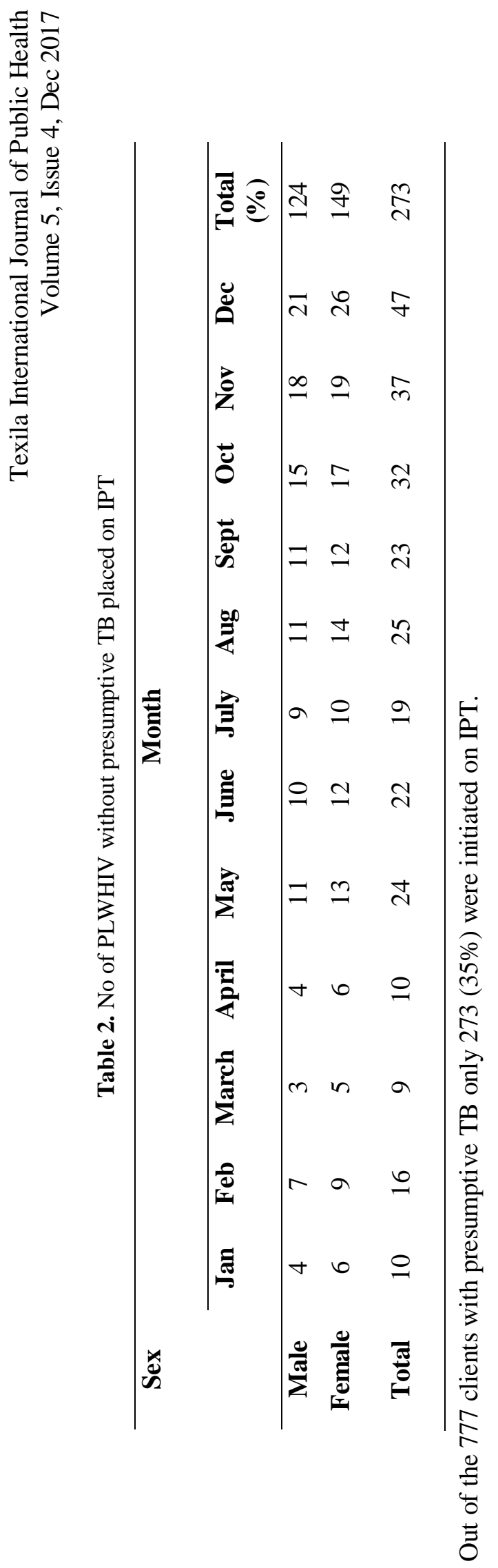


DOI: $10.21522 / \mathrm{TIJPH} .2013 .05 .04 . \mathrm{Art} 022$

ISSN: $2520-3134$

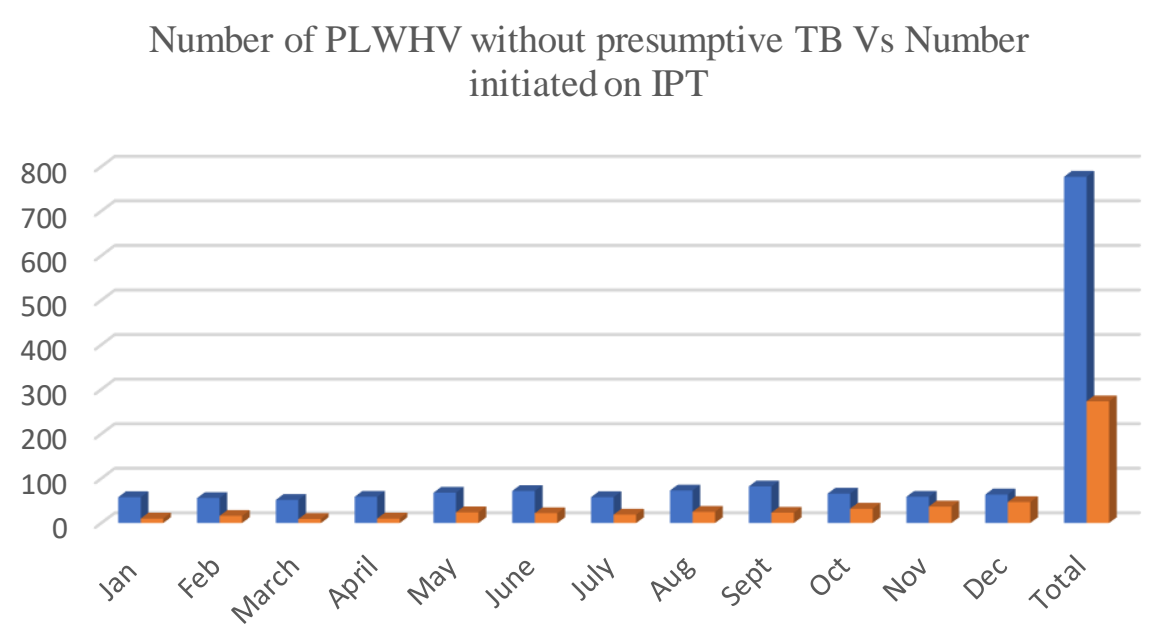

number without presumptive TB $\square$ Number without presumptive TB initiated on IPT

Figure.2. Number of newly enrolled clients screened for TB without presumptive TB Vs number initiated on IPT

Table 3. HIV-infected patients on treatment who completed isoniazid preventive therapy vs. HIV-infected patients on treatment who did not complete isoniazid preventive therapy

\begin{tabular}{lll}
\hline Characteristics & Completed IPT & Did not complete IPT \\
\hline $\begin{array}{l}\text { Receiving HAART at time of } \\
\text { IPT }\end{array}$ & $143(93 \%)$ & $10(7 \%)$ \\
$\begin{array}{l}\text { Not receiving HAART at } \\
\text { time of IPT }\end{array}$ & $97(80 \%)$ & $23(20 \%)$ \\
\hline
\end{tabular}

A higher frequency of adherence to IPT was seen among patients on HAART; $93 \%$ of the clients receiving HAART at the time of IPT completed their IPT, while $7 \%$ did not completed their IPT.

\section{Discussion}

This study showed that even though all the clients enrolled in to HIV were screened for TB, less than half $35 \%$ clients without presumptive TB were initiated on IPT. Also reported was that a higher proportion $(93 \%)$ of the clients receiving HAART at the time of IPT completed their IPT, while a lower proportion (80\%) of the clients not receiving HAART at the time of IPT completed IPT. This indicates that adherence to IPT is higher among the patients on HAART compared to those who did not commence HAART.

The finding of this study is similar to a study conducted in THrio Brazil that shows IPT completion was higher among HIV-infected patients receiving HAART (87\%) than those not yet receiving HAART (79\%, $P<0.01$ ). (Durovni et al. 2011). In another study conducted in Nigeria, reports showed that IPT was being utilized for prevention of TB in HIV/AIDs though at a lower rate than what was reported in this research. (Nwokeukwu H I, et al., 2015). Another study conducted in Kampala Uganda shows that widowed, separated, or divorced HIV clients were found to be associated with a higher risk of loss to follow-up. (Namuwenge P M. et al. 2011). In a review of 41 articles published, reports showed that provision of IPT to PLWHIV to reduce TB-associated morbidity and help to highlight the need to strengthen IPT implementation. IPT should be provided to all PLHIV without presumptive TB because of it benefit. (Briggs M A. et al. 2015).

The sex distribution also showed that the uptake of IPT was more with the females. Reasons for this may be due to the fact that females have been reported to have a better health seeking behavior than males, thus they may then to comply more with their medication than male patients. 
The finding of this study also suggests the need to improve the initial adherence counseling and ensures client understands the benefits of commencing IPT. Some of the possible reasons for not completing the IPT may include: adverse drug reactions due to isoniazid, inconsistent IPT supplies, people enrolled on IPT are a relatively healthy people who may not see the need of IPT over period of six months and some health care workers may not understand the benefits of IPT due to knowledge gap, and therefore may not adequately encourage patients to be compliant to IPT.

There was improvement in the number of clients that were placed on IPT, because in the first three quarter of the year 2015 only 157 clients were initiated on IPT, but within the last quarter of 2015 (October -December 2015) the number increased to 273. This showed the percentage uptake improved in the last quarter, although much need to be done by making sure that Doctor and other health care workers are reminded to identify those who are eligible and initiate them on IPT. With this achievement, there is possibility of more patients to be place on IPT, as more awareness and capacity building on IPT implementation are being intensified among the Health care workers.

\section{Conclusion}

Isoniazid preventive therapy is effective and significantly reduces the occurrence of tuberculosis infection among HIV infected individuals. However, it utilization in the prevention of TB among the PLWHIV in this health facility is very low, however adequate supply of Isoniazid, creation of more awareness among health care providers and Patients, will improve uptake of IPT among PLWHIV. Effective and continues adherence counselling to eligible PLWHIVs before the commencement of IPT, especially the Pre-ART clients will help in reducing the rate of defaulters to IPT.

\section{Recommendations}

- Government to ensure increase availability and access to isoniazid tablets across all the hospitals providing HIV prevention, support, care and treatment services.

- Effective adherence counselling to eligible PLHIVs before the commencement of IPT, will help in reducing the rate of defaulters to IPT.

- Family and community members should be involved as patient treatment supporters in taking prescribed Isoniazid medication.

- Conduct more studies across the state on the IPT implementation among the PLWHIV.

- Doctors/Health care worker's capacity need to be built on the IPT service implementation.

- Government to strengthen IPT as an integral part of TB/HIV planning, reporting and monitoring and evaluation at both secondary and tertiary hospital.

\section{Acknowledgement}

We would like to acknowledge the management and entire staff of the Anti-retroviral unit of Specialist hospital Yola for their numerous contribution. We would also like to acknowledge Pharmacist Zira Mathias for the support and guidance during the period of this study.

\section{References}

[1]. Assebe L F, Hailemariam L R, Alem D W, Wondwossen T L and Saba M L. (2015). The effect of isoniazid preventive therapy on incidence of tuberculosis among HIV-infected clients under pre-ART care, Jimma, Ethiopia: A retrospective cohort study. BMC Public Health. 15:346. DOI 10.1186/s12889-015-1719-0.

[2]. Briggs MA et al. (2015) Use of isoniazid preventive therapy for tuberculosis prophylaxis among people living with HIV/AIDS: a review of the literature Journal of Acquired Immune Deficiency Syndrome; 68 Suppl 3:S297-305.

[3]. Chehab J.C, Vilakazi-Nhlapo K, Vranken P, Peters A, Klausner J D. (2011). Implementation of Isoniazid Preventive Therapy (IPT) in South Africa, 2011.

[4]. Durovni et al. (2011). The implementation of isoniazid preventive therapy in HIV clinics: the experience from the TB/HIV in Rio (THRio) Study AIDS. Author manuscript; available in PMC 2011 March 29. 
DOI: $10.21522 / \mathrm{TIJPH} .2013 .05 .04 . \mathrm{Art} 022$

ISSN: $2520-3134$

[5]. Denegetu and Dolamo (2014). Tuberculosis case finding and isoniazid preventive therapy among people living with HIV at public health facilities of Addis Ababa, Ethiopia: BMC Public Health 2014, 14:52.

[6]. Federal Ministry of Health (FMOH) Department of Public Health. Guidelines for Clinical Management of TB/HIV related conditions In Nigeria 2nd edition, 2008. http://www.who.int/hiv/pub/guidelines/nigeria.pdf.

[7]. Granich, R, Akolo, C, Gunneberg, C, Getahun, H, Williams, P and Williams, B. 2010. Prevention of Tuberculosis in People Living with HIV. Clinical Infectious Diseases 50 (Suppl 3):S215-S222.

[8]. Howard and El-Sadr. (2010). Integration of Tuberculosis and HIV Services in Sub-Saharan Africa: Lessons Learned. Clinical Infectious Diseases 2010; 50(S3):S238-S244.

[9]. Melissa A. B, Courtney E, Surbhi M, Nicholas K T, et al. (2015). Use of Isoniazid Preventive Therapy for Tuberculosis Prophylaxis among People Living With HIV/AIDS. Journal of Acquired Immune Deficiency Syndromes; 68: S297-S305.

[10]. Margaret Olabisi Araoye. 2004. Research Methodology with statistics for health and social sciences. page 117120.

[11]. Namuwenge PM, et al. (2011). Loss to follow up from isoniazid preventive therapy among adults attending HIV voluntary counseling and testing sites in Uganda. Trans $R$ Soc Trop Med Hyg (2011), doi:10.1016/j.trstmh.2011.10.015.

[12]. Nwokeukwu H I, Okorie O, Emma-U U, Ukegbu A, Nwogu K1, Ajuogu E1, Osunkwo D, Asinobi A. (2015). Use of Isoniazid Preventive Therapy on HIV/AIDS Patient in a Tertiary Health Facility South Eastern Nigeria. Science Journal of Public Health 2015; 3(2): 265-268 (http://www.sciencepublishinggroup.com/j/sjph) doi: 10.11648/j.sjph.20150302.26.

[13]. The Global Fund to Fight AIDS, TB and Malaria (GFATM): Collaborative TB/HIV activities information Note. http://www.theglobalfund.org/documents/rounds/11/R11_TB/HIV_InfoNote_en/(accessed 21 October 2012).

[14]. United Nations for AIDS Program (UNAIDS): Global Fund Information Note: TB/HIV Collaborative Activities. http://www.stoptb.org/assets/2012/02/21/global/ TBHIV Collaborative Activities (accessed 2 January 2013).

[15]. World Health Organization. Global tuberculosis report 2015. Geneva, Switzerland: WHO; 2015. WHO/HTM/TB/2015.22. www.who.int/tb/publications/global_report/en Accessed June 2016.

[16]. World Health Organization. 2007. Treatment of TB: guideline for national programmers. $3^{\text {rd }}$ edition. World Health Organization, Geneva. WHO press.

[17]. World Health Organization (WHO). Guidelines for Intensified Case-Finding and Isoniazid Preventive Therapy for People Living with HIV in Resource-Constrained Settings. Geneva, Switzerland: WHO; 2011.

[18]. World Health Organization (WHO). Global tuberculosis report 2013. Geneva, Switzerland: WHO; Oct 2013. http://apps.who.int/iris/bitstream/10665/91355/1/9789241564656_eng.pdf.

[19]. World Health Organization (WHO): Global Tuberculosis Control. 2011, http://www.who.int/tb/2012/03/23/publications/global_report (accessed 31 December 2012), Report Google Scholar. 